\title{
The Ultimatum Game: An Introduction to Quantitative Literacy in a Social Justice Context
}

Robert G. Root

LafayetteCollege, robroot@lafayette.edu

Follow this and additional works at: https://digitalcommons.usf.edu/numeracy

Part of the Behavioral Economics Commons, Language and Literacy Education Commons, Other Mathematics Commons, Scholarship of Teaching and Learning Commons, Science and Mathematics Education Commons, and the Social and Cultural Anthropology Commons

\section{Recommended Citation}

Root, Robert G.. "The Ultimatum Game: An Introduction to Quantitative Literacy in a Social Justice Context." Numeracy 12, Iss. 2 (2019): Article 4. DOI: https://doi.org/10.5038/1936-4660.12.2.4 


\title{
The Ultimatum Game: An Introduction to Quantitative Literacy in a Social Justice Context
}

\author{
Abstract \\ The Ultimatum Game is a two-person, multiple-strategy game widely used in the experimental social \\ sciences to demonstrate the human propensity for costly punishment in response to inequitable \\ treatment. The game serves to provide quantitative evidence for a diversity of fairness norms across \\ cultures. The play of the game and its interpretation offer nuanced views of the nature and importance of \\ quantitative literacy. Its use in a writing seminar connecting quantitative literacy and social justice is \\ described.

\section{Keywords} \\ game theory, sub-game perfect equilibrium, fairness norms

\section{Creative Commons License} \\ (c) (i) (8) \\ This work is licensed under a Creative Commons Attribution-Noncommercial 4.0 License

\section{Cover Page Footnote} \\ Robert G. Root is professor and former head of the Department of Mathematics at Lafayette College in \\ Easton, PA. He is currently serving as the Clerk of the Faculty at Lafayette. He enjoys reading Numeracy, \\ this is his second contribution to the journal.
}




\section{Introduction to the Ultimatum Game}

Game theory is a branch of mathematics that models social interactions, and so makes an ideal topic for quantitative literacy pedagogy. In fact, Rick Gillman and David Housman have a textbook designed specifically to teach quantitative literacy skills using game theory as the unifying theme (Gillman and Housman 2009).

While the text begins with combinatorial games, this article describes the use of a classical two-person, multiple-strategy game, the Ultimatum Game, as an introduction to quantitative literacy in a social justice context. This introduction occurs in the first week, starting on the first day, of a first-year writing seminar called "The Mathematics of Social Justice." As a writing seminar, the class is small (capped at 16 students), and is not expected to present any mathematics at all. The course seeks to make a number of connections between quantitative literacy and social justice, with one central idea being that game theory can demonstrate that a society perceived by its members as just is a robust society, able to overcome obstacles because individual members are willing to make sacrifices in order to perpetuate its just nature.

The class draws students from a wide variety of backgrounds and generally includes students with interests in mathematics (or its traditional client disciplines like engineering or the sciences) as well as students who are primarily interested in social justice, with little enthusiasm for mathematics. Over seven repeated offerings, the vast majority of students have found the Ultimatum Game an engaging first-day-of-college experience.

The Ultimatum Game is not as well-known as the Prisoners' Dilemma, but it holds a special place in the pantheon of game theory. For example, the Canadian behavioral economist Yoram Halevy has given multiple presentations with the title "Is the Ultimatum Game the Ultimate Experiment?" (see, for example, Halvey and Peters 2007), and William Poundstone, in his readable and informative introduction to the Ultimatum Game and its role in pricing theory says, "The ultimatum game is claimed to be one of the most frequently performed of all human experiments today" (Poundstone 2010, 114). Game theoretician Martin Nowak and coauthors claim "The Ultimatum Game is quickly catching up with the Prisoner's Dilemma as a prime showpiece of apparently irrational behavior" (Nowak et al.2000, 1773). The game is prominent in experimental economics, social psychology, and cultural anthropology, as well as among game theorists.

The game has unique value in teaching quantitative literacy in a social justice context. It offers quantitative evidence of the concrete value humans place on equitable treatment, a foundation for social justice. It also offers an early glimpse 
at the richness of quantitative literacy and its potential implications and limitations. To appreciate the evidence for these assertions, we need to understand how the game is played.

The two players have different roles: one is the "proposer" and the other is the "responder." They are provided with a "stake," usually some money that can be split between them. In the author's classroom, the stake is ten one-dollar bills, so that there are only eleven possible splits: "\$0 for you, $\$ 10$ for me," "\$1 for you, $\$ 9$ for me," and so on, up to "\$10 for you, $\$ 0$ for me." The proposer proposes a split of the stake, and the responder indicates which splits are acceptable and which are not. If the responder accepts the proposal, the game is won and the stake is awarded to the two players split according to the proposal. If the responder does not accept the proposal, the game is lost and the players get nothing. There is no further negotiation: a proposal is made and either accepted or rejected; hence the name "Ultimatum Game." The decision tree for the game is shown in Figure 1.

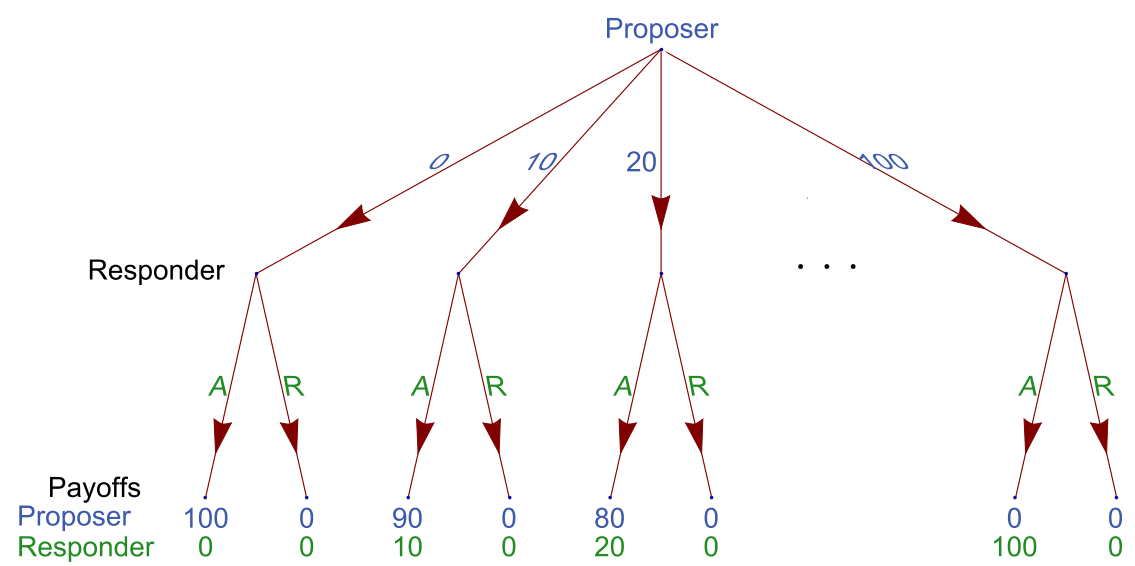

Figure 1. Decision tree for the ultimatum game with a general stake divided into $10 \%$ increments. First the proposer chooses a proposal, which is denoted by the percent of the stake going to the responder. Then the responder chooses to accept or reject the proposal. The outcome is the payoff for the players, indicated as an ordered pair, with the proposer's payoff given first. Because the responder does not have to choose to accept or reject until the proposal is known, a complete strategy for the responder includes a response for every possible proposal.

Let us assume that the payoff of the game is the share of the $\$ 10$ stake that each player will receive and apply elementary game theory. Here and in the balance of the article we will use feminine pronouns for proposer and masculine for responder. (A reader unfamiliar with game theory may safely skip this paragraph and the next 
two. Pick up with the paragraph at the top of page 4 beginning "One does not need to be a master of game theory ....") To start, we notice that while the proposer has only 11 possible strategies corresponding to the 11 proposals, the responder has $2^{11}$ $=2,048$ possible strategies, as each proposal can be accepted or rejected independently. As a result, the game has just as many Nash equilibria. Each can be characterized by the responder accepting the proposer's offer, but no proposals that are more favorable to the proposer. The responder can change his action in response to proposals more generous to him without providing the proposer with way to improve her payoff. That is, any strategy for the responder in which at least one proposal is accepted is part of a Nash equilibrium when coupled with the proposal most favorable to the proposer that is acceptable to the responder. This describes 2,047 Nash equilibria (in pure strategies), with the responder's last strategy of not accepting any proposals having an equilibrium proposal that offers nothing to the proposer.

Two of these Nash equilibria are sub-game perfect. They both occur when the responder accepts any strategy that offers him a positive split, and the proposer maximizes her payoff. A sub-game perfect strategy profile has optimal play not just on the entire game tree, but on every proper sub-game tree, obtained by looking at and below the decision nodes of the responder. Looking at Figure 1, each of the eleven decision nodes for the responder is the root of a sub-game tree, and for all but the leftmost, the responder's payoff is maximized by accepting the proposal. Thus, the responder's sub-game perfect strategy must include accepting all of these proposals. The exception is the proposal in which the responder receives nothing; here the responder is indifferent between accepting and rejecting.

Thus, the strategy profile when the responder accepts all proposals and the proposer chooses to take all the money is a sub-game perfect equilibrium. So is the strategy profile in which the responder accepts all proposals except the one that offers him nothing, and the proposer proposes offering $10 \%$ to the responder and keeping $90 \%$ for herself, the minimum positive offer. The first of these is not strict, however, as the responder is indifferent between accepting and rejecting the proposal that is made. Thus, the responder could credibly reject that proposal. The responder's expected payoff can decrease all the way down to zero as the probability of rejection of the equilibrium proposal increases, with no change in the responder's payoff of $\$ 0$. The second equilibrium is strict; the proposer's payoff does not decrease at all if the responder chooses to alter the probability of accepting the greediest proposal. This strict sub-game perfect equilibrium is the game theoretic optimal solution for the game, under the assumption that the payoffs are comprised solely of the portion of the stake each player receives. 
One does not need to be a master of game theory to see that the responder gets more money by accepting any positive offer than by rejecting it and that the proposer maximizes her share of the stake by offering the minimum positive share $(\$ 1$ in the case of the stake of ten one dollar bills) to the responder. This line of reasoning is easy to follow, and it leads to the strict sub-game perfect equilibrium solution to the game. (For readers who have skipped the preceding three paragraphs, the italicized phrase is just a game theoretic term for a way to optimize play of the game for both players. Readers wishing a more thorough definition of the term can consult any introductory game theory text, for example, Schecter and Gintis 2016.) However, this rational conclusion is confounded by practice. In my classroom, most proposers offer even or nearly even splits and most responders reject proposals that greatly favor the proposer. Thus we arrive at a seeming paradox: human behavior is at odds with the predictions of this mathematical model.

This leaves us with two questions:

1) Do humans more generally behave this way and if so why?

2) How can this paradox be useful in teaching quantitative literacy?

The rest of this article is an attempt to answer these questions. With the reader's indulgence, let's consider them in order.

\section{Ultimatum Game as Experiment}

As a math problem, the Ultimatum Game appears to be a breeze. One could justify the significance of a sub-game perfect equilibrium as a solution to a game with the colloquial reasoning offered at the end of the previous section. The problem is not that the author's classroom is in some way unique; the only known population of humans that plays the Ultimatum Game using the optimal strategy just deduced are the Machiguenga of eastern Peru, among whom "[c]ooperation above the family level is almost unknown" (Henrich 2000, 974). Poundstone (2010) adds that the only people who behave according to economists' postulates themselves lived in a society with no economy to speak of.

There has been an enormous array of studies of the way that different cultures around the world play the Ultimatum Game. The class has been assigned to read the most significant of these, the study of 15 micro-cultures conducted by a large team of anthropologists, led by Joseph Henrich (Henrich et al. 2006). The study provided a stake of roughly a day's wages to players in different groups. The players were kept anonymous from one another in order to eliminate considerations of "kinship, reciprocity, reputation, or the immediate threat of punishment" from 
affecting players' decisions (p. 1768). Under carefully controlled conditions, and with instructions that were painstakingly translated to the local native language, the anthropologists strove to create an experiment that was as uniform across locations as possible. The results are shown in Figure 2.

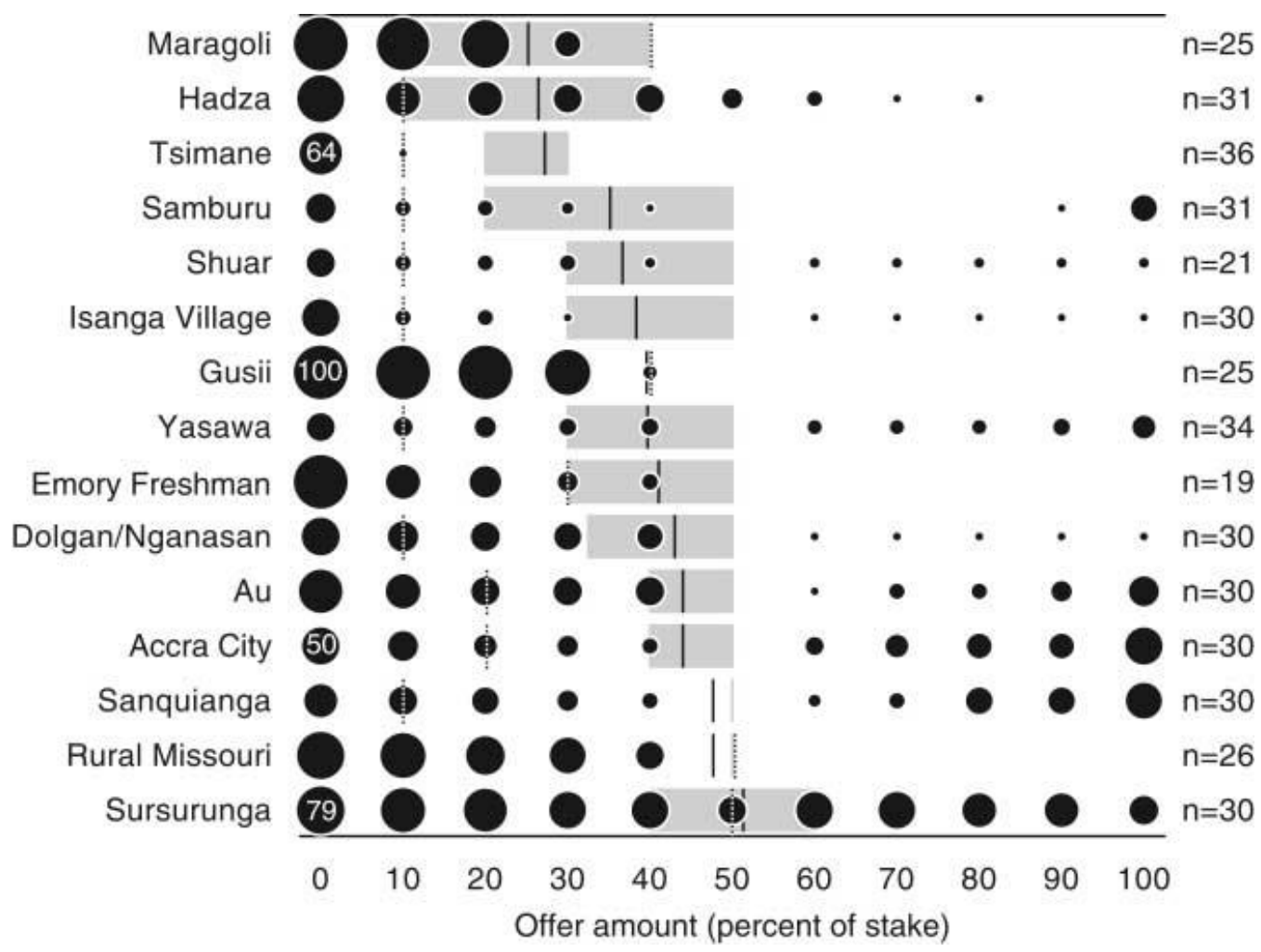

Figure 2. Figure 1 from Henrich et al. (Henrich et al. 2006, p. 1767), with results of the ultimatum game played in different micro-cultures. The numbers on the horizontal axis denote proposals by the percent offered to the responder, and each row corresponds to a different group, with the name on the left and the number of games played on the right. The area of the black disks indicates the proportion of responders rejecting the proposal in the indicated group. The gray rectangles indicate the span of the middle $50 \%$ of proposals, and the black vertical bars indicate the mean proposal. The dashed vertical bars represent the income-maximizing offer, the proposal with the largest expected monetary payoff given the strategies of responders in that group. Figure reused by permission.

The meanings of the components of the plot are described in the caption, but interpreting the content of the plot is an extended undertaking, occupying considerable class time. Here is a slightly more detailed description than one class period has allowed. First, to aid us in understanding the relative magnitudes of the black disks, note that a few of them contain numerals indicating the percent of 
responders represented by that disk. So, for example, $100 \%$ of responders in Gusii (a group from Kenya) refused the proposal where the responder gets no money. The differences in the rows of disks represents a wide diversity of behaviors by responders. Notice that there are at least four locations with the same responder behavior that is exhibited in the author's classroom, as described at the end of the Introduction.

Among the Maragoli (a different group in Kenya), Gusii, Emory Freshmen, Rural Missouri, and Dolgan/Nganasan (a Siberian group) responders more or less strongly reject the proposals in which they receive substantially less than proposers. All other proposals are accepted by all (or nearly all in the case of the Dolgan/Nganasan) responders. Let's refer to these groups as cultural collection A.

Compare these with the Tsimane (a Bolivian group), Samburu (a third Kenyan group), Shuar (Ecuadoran), Isanga Village (Tanzanian), and Yasawa (from the eponymous island in the Fiji archipelago). Responders in these latter groups generally accept most proposals, with the possible exception of the proposal in which the responder gets nothing. This will be cultural collection B.

Cultural collection $\mathrm{C}$ consists of $\mathrm{Au}$ (from Papua New Guinea), Accra City (the capital city of Ghana), and Sanquianga (a group from the Pacific coast of Colombia). Note that responders in these groups reject proposals with payoffs that are far from equal for responder and proposer in either direction. Following the notation of the article, we will refer to proposals that offer responder more than proposer as hyperfair.

This leaves only the Hadza (foragers from Tanzania) and Sursurunga (from Papua New Guinea) with distinctive patterns. The Hadza seem to include decreasingly prevalent responders who expect increasingly hyperfair proposals. The Sursurunga include responders who reject every proposal in substantial proportions.

We turn now to consider the play of the proposers. In the figure, the groups are arranged in order of increasing mean proposal's share for the responder. That is, the black vertical bars marking the mean proposal move to the right as we go down the rows of the graph. It is not surprising that the groups in collection B tend to be toward the top of the figure. Generally aware that the responders are unlikely to reject greedy proposals, proposers in these groups are likely to make them. What is surprising is the relative generosity of the proposals that are made. For all the groups in collection $\mathrm{B}$, the income maximizing proposal ${ }^{1}$ is the minimum positive

\footnotetext{
${ }^{1}$ The income for any proposal is $p * r$ where $p$ is the value of proposer's fraction of the stake and $r$ is the fraction of all responders accepting the proposal. In probabilistic terms, income is the expected value of the payoff for making the proposal against a randomly chosen responder.
} 
offer to responder. However, even among the Tsimane, whose proposers are the stingiest, at least $75 \%$ of proposers offer responders at least $20 \%$ of the stake. And this is to responders who hardly reject any positive offer. The other groups in collection B have noticeably more generous proposals, as well as a few more responders more willing to reject inequitable offers. We could characterize the general reaction to the game in these groups as, "let's make sure someone takes the anthropologist's money!" The responders generally are playing the sub-game perfect equilibrium strategy, but the proposers generally are making offers that are fairer than the responders require.

In collection A, the Gusii stand out as the group with the clearest mutual understanding for dealing with the game. A large majority of responders reject all proposals in which they get less than $40 \%$ of the stake and accept all more generous proposals. A large majority of proposers offer responders $40 \%$ of the stake. Everyone seems to be on the same page in this group. Rural Missouri has much the same behavior but with a 50-50 proposal $^{2}$ being the convention and with either a few more proposers making less generous proposals or the proposers who vary from the convention vary by being greedier. (This is visible because the mean in this group is further from the widely agreed-upon proposal than for the Gusii.)

The most genuinely puzzling group in the study is the Maragoli. Just as among the Gusii, the income maximizing proposal is to offer the responder $40 \%$ of the stake, but the actual proposals are the least generous of any group. Given the strong uniformity of the responders, it is unusual that most proposers are unaware or uncaring that their proposals are very likely to be rejected. We can only speculate on the motivations in this group. One possible explanation is that displays of aggressiveness are more valuable than the stake. It might be worth more to be seen as jealously protecting one's own primacy in the negotiation than to actually win it. In any case, the Maragoli are remarkable for the rate at which the game was lost.

The two other groups in collection A include some proposers who make proposals that are likely to be rejected but seem to generally identify either a 40-60 or 50-50 split as the convention.

Collection $\mathrm{C}$ groups have responders who tend to offer roughly even splits, which is consistent with the expectations of the responders. Even the Sanquianga, whose responders are so generally accepting of all proposals that this group might belong in collection $\mathrm{B}$, have proposers highly sensitive to the convention that a 5050 split is the only one universally acceptable. The archetype for collection $\mathrm{C}$ might be derived from Ghanaian culture, where Accra City is located. Here is a passage

${ }^{2}$ When identifying proposals in this way, we will give the percentage of the responder first and proposer second. In this case that is immaterial, but it will not always be so. 
from a blog of an American serving in an NGO in Ghana. She describes how Ghanaians often would solicit gifts from her, like the title of the blog entry, "Give me your camera." She describes the situation as follows.

We were told that when someone asks us for our things it is because of one of two thingsor possibly a combination of both. The question might simply be a way to start a conversation with a stranger. Or it might be part of a more complex system of gift giving at the center of relationships in this part of the world. Specifically, a person might just want to be your friend. If you give that person something, they are indebted to you, must give you a gift in return somewhere down the line, and thus begins a beautiful friendship. We were told in our cultural training that Ghanaians don't expect to actually be given the thing, but will happily take it if you decide to give it (Webber 2017).

So, gifts in Ghana come with a clear expectation of reciprocation. While a gift from an American woman seemed like an overture to a highly desirable friendship, it seems reasonable to assume that a gift from an unknown Ghanaian stranger is a much more risky proposition. It is likely that responders' rejections of favorable proposals are intended to avoid obligations to strangers. This may also explain the prevalence of 50-50 splits among proposers, lest by taking a greater share they feel an obligation to their responders. According to Poundstone $(2010,123)$, the $\mathrm{Au}$ have similar social norms, in which gifts "create an obligation to reciprocate, and most people would prefer not to have that burden." Similar cultural norms might explain the behavior in the Sanquianga, as well. Concerning this Colombian group, Cardenas quotes Price as saying, "Any individual can relate the names of many person[s] to whom at one time or another he has given food; some of these have eventually reciprocated, while the others may be called on when the need arises." (Cardenas 2014, 418; Price 1955, 20)

We turn now to the last two groups, which do not easily fit into any of the collections. The Hadza proposers are nearly as greedy as those of the Maragoli and seem unaware of or unconcerned by the presence of responders who expect more than half the stake. These responders seem to be in the minority, and the figure (and the article) shows no evidence that any proposer made a hyperfair offer. It is possible that the Hadza have motivations similar to the Maragoli, with displays of aggressiveness by both responders and proposers. Another similarity is the frequency with which the Hadza subjects lost the game.

This is also true for the Sursurunga, although the proposers in this group made the most generous proposals on average of any group, and this group's average proposal is the only one to exceed 50-50. The difficulty seems to be the pattern of the responders, with a sizable fraction rejecting every possible proposal. No other group exhibits this behavior. One possibility is that the Sursurunga include a fraction of responders that see themselves in a position to expect hyperfair offers, 
as among the Hadza. The balance of the Hadza seem to be playing like culture collection B, allowing any proposal, except possibly one in which they receive nothing. In this interpretation, the balance of the Sursurunga play like collection C, punishing hyperfair and greedy offers in roughly equal measure. In contrast to the Hadza, the proposers from the Sursurunga show some evidence to support this claim, since the middle $50 \%$ of proposals include hyperfair $60-40$ proposals, a behavior shown by no other group. This is a third group in which the game appears to be lost frequently.

Having devoted considerable effort to examining the results of Henrich, et al.'s (2006) magisterial study of the play of the Ultimatum Game across fifteen cultures, we consider the play of students in the author's classroom, summarized in Figure 3 using the same representation of the results as in Figure 2.

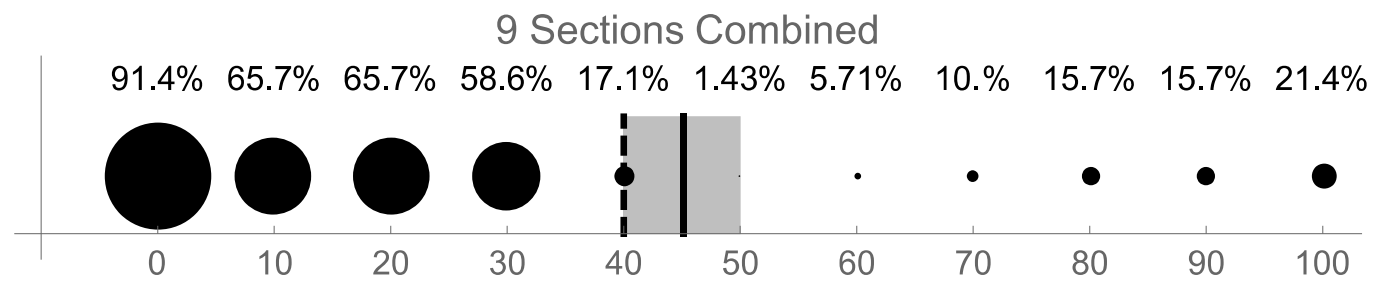

Figure 3. Plot for results of the Ultimatum Game as played in the author's classes. This plot uses the same conventions as Figure 2 and combines results over nine sections. It includes data for 70 responders and 72 proposers.

While the data from classroom activities cannot be seriously compared with that collected in carefully designed experiments, the results are suggestive. We see behavior that is similar to collection A, an unsurprising result since the two groups from the US examined by Henrich et al. (2006) also belonged to this collection. The data includes five minimum positive proposals (10-90), or about $7 \%$ of all proposals were the sub-game perfect equilibrium strategy. It is remarkable that in a course with "mathematics" in the title, more students didn't work out and implement optimal play as we have described it. We can see that the data predicts a success rate of about one third for such proposals; in practice only 1 (so one fifth) was accepted by the responder.

One other aspect of the game that arises in classroom discussions is the effect on play of the size of the stake. Other experimenters have examined this matter, with Lisa Cameron (1999) conducting experiments in Indonesia, Robert Slonim and Alvin Roth (1998) in the Slovak Republic, and Steffen Andersen et al. (2011) in rural northern India. The Cameron (1999) study used the relatively low income levels of Indonesia to raise the stakes. The maximum stake of roughly $\$ 100$ (US in 
1994) was about 3 months of expenditures for participants in the study. The results suggested that while responders marginally increased their likelihood of accepting proposals offering smaller proportions of the stake, the distribution of proposals made did not change as the stake increased.

Slonim and Roth (1998) were able to reproduce this result but also offered the players the chance to gain experience and found that as proposers learned that they could successfully make less generous proposals when the stakes were large, they applied this knowledge to their play.

Andersen et al. $(2011,3428)$ noted that "proposers stubbornly insist on offering nontrivial fractions of the pie when playing the game ..." To deal with this difficulty, "...by use of specific language in the experimental instructions, [the experimenters'] design successfully elicits low offers from proposers over all stakes levels, making it possible to measure stake effects over large stakes changes." This experiment begins by explicitly informing the proposers, “...if the responder's goal is to earn as much money as possible from the experiment, he/she should accept any offer that gives him/her positive earnings, no matter how low" (Andersen et al. 2011, 3430). Having offered this explicitly monetary frame to guide the proposers' thinking, the experimenters were able to demonstrate that larger stakes resulted in offers of smaller fractions of the total stake, although the absolute size of the portion of the stake offered grew with the size of the stake.

It is remarkable that across the range of locations, players were unlikely to adopt the naive assumption that "the responder's goal is to earn as much money as possible" unless that assumption was offered to them explicitly. This behavior is consistent with the idea that most players place value on equitable treatment. In Cameron (1999) and Slonim and Roth (1998), the perceived value of equitable treatment seems to scale roughly, though not exactly, with the size of the stake. The Andersen et al. (2011) study, which explicitly suggested a monetary approach in the instructions, still suggests the more plausible scenario that the value placed on equitable treatment does increase with the size of the stake but not proportionally.

While the author finds these results helpful in guiding discussion of the Ultimatum Game in class, the studies themselves have not been part of the assigned reading for the class. Having reviewed them, we are now in a position to answer the first question posed about human behavior in the Ultimatum Game: People play the game in an unusual variety of ways and only extremely rarely in the way postulated by economists as optimal. As for why this variety of ways of playing exists, it seems to reflect different conceptions of fairness, and thus suggests that the game can tell us a great deal about social justice. 


\section{Ultimatum Game as Introduction to Social Justice}

The course syllabus offers a definition of social justice that is a variant of that given in the "Social Justice" entry in the Encyclopedia of Community (Erickson 2003, 1309-14): "Social justice is fairness in the creation and distribution of benefits and burdens that derive from living together in a society." This definition covers most of the wide range of social justice issues that concern people today: the ability to vote, marry, receive equal pay for equal work; the delivery of education and health care; the possibilities of where one might live or work and how one might worship; the decision to go to war or sue for peace; all of these are either benefits or burdens that inevitably entail social choices. The definition does not call out potentially oppressed classes of people, like racial or ethnic minorities, gender or religious identities, sexual orientations, national origin, age, or disability. Instead, the definition hinges on the word "fairness."

The Ultimatum Game is a lens for examining fairness, bringing into focus the fact that while the understanding of equitable treatment varies somewhat within cultures, it varies radically across cultures. In the previous section we learned that only the Machiguenga, for whom the family group seems to constitute the extent of societal cooperation, appear to place no importance on equitable treatment of an anonymous stranger (Henrich 2000). All other groups show evidence that there is an understanding of equitable treatment implicit in this game that was designed to be an abstraction from daily living. That is, despite the foreign nature of the game, most groups have players that recognize in it an equitable way to play, and they are generally successful at playing it. The notable exceptions are the Maragoli, the Hadza and the Sursurunga, discussed above. The observation that some societies have difficulty recognizing a convention for fair behavior in the Ultimatum Game supports the assertion that there are a variety of conventions for fairness across human societies. The play of the Sursurunga suggests that the game might evoke two competing fairness norms in their culture.

Beyond this, the game illustrates that humans routinely make tangible monetary sacrifices to enforce the norms for fairness that exist in their society. The literature reviewed makes a great deal of what is called costly punishment, when the responder chooses to punish proposals he deems unfair at the cost of receiving nothing himself. However, the groups in collection B are characterized by proposers who routinely make offers that are more equitable than they need to be, given the behavior of the responders. It is likely that at least in many cases, proposers are giving away money that they might have kept for themselves, which represents a personal sacrifice to enforce a fairness norm distinct from costly 
punishment. In results so recent that they have never been used in my class, Candelo et al. (2019) offer evidence that this behavior is not simply risk aversion, and might be genuinely altruistic.

The results of the experiments varying the sizes of stakes suggest that these fairness norms are robust even as the size of the stake increases but that they are only norms and that offering players an alternative view of the nature of the game and the "fair" way to play can override these norms.

Thus, the game demonstrates that conceptions of fairness are not universal, but different societies seem to enforce their own understanding of fairness in different ways. Certainly this enforcement is also not universal, but it is widespread in most societies, and the classroom activity usually brings home to the class the value of recognizing the social norm in our society and the misfortune of those who fail to recognize it.

The irony is that the naive model for the game described earlier, and often observed by students, leads to this failure of recognition. Thus, we find ourselves facing the second question posed in the Introduction but even more bleakly: why use this game as an introduction to quantitative literacy if the outcome is likely to punish the students who are able to deduce a simple underlying quantitative structure to the game?

\section{Ultimatum Game as Introduction to Quantitative Literacy}

\section{The Primacy of Modeling}

This game was created to be mathematically modeled. When, in 1977 or '78, Werner Güth and his colleagues developed the game, it was intended as a tool to investigate human behavior and how behavior deviated from the classical economic model, epitomized by our game theoretic analysis (Güth et al. 1982). According to Poundstone (2010, 113), "Güth said it was never his intention to demonstrate that humans don't behave as economists assumed. 'That would have been overkilling an already dead man.' He was interested in devising 'the easiest nontrivial ultimatum bargaining games with only two players and seeing how real people would play them." When a student falls into the trap of the classical model, there is a learning opportunity that can benefit the entire class.

It is critically important that students understand that mathematical reasoning is pointless unless there is reason to believe that underlying assumptions capture the essence of the situation being modeled. When the student who has identified 
the sub-game perfect equilibrium loses, the stigma of losing can be softened considerably by the instructor acknowledging the student's cleverness while also acknowledging the loss as a learning opportunity. (In the rare instance when the equilibrium proposal is accepted, the responder is a loser; most other responders in the classroom receive much more generous results from their play. The hardest person to convince is a responder who plays the sub-game perfect strategy but receives a generous proposal.)

From the record of the actual play of the game, we can revise the naive model, which assumes that the stake is the only source of value to account for in play. The data demonstrate that the stake cannot be the only source of value in the game, and our payoff must include another source of value, equitable treatment, which most players' behavior demonstrates carries value comparable to the stake, or a portion of it. Specifically, in the classroom, and in many cultures around the world, responders are willing to turn down money in order to punish a proposer that they perceive as treating them unfairly. The exact nature of unfair treatment depends on the culture, as we have seen, but in many groups (all groups in collections A and C) responders are commonly willing to sacrifice winnings in order to punish a proposer perceived as unfair; behavior we have learned is called costly punishment.

Individual responders have different values assigned to the various payoffs based on how they value the monetary winnings and the value that they place on their perception of the fairness of the proposal. When this is taken into account, responders should accept proposals where the monetary value exceeds any penalty for perceived unfairness. It is tempting to offer an equation where the value $v$ of a proposal is dependent of the fraction of the stake $f s$ less the penalty $p$ for the degree of inequity the responder perceives in the proposal: $v=f s-p$. This implies that the perception of inequity and the value of the stake are static and comparable in a continuing way on a common scale. This assumption is dubious; it is more likely that the penalty in particular (and to some extent the subjective valuation of the money in the stake) vary over time, and even classroom discussion as the game unfolds can alter the decisions of responders and proposers alike.

In our revised model, proposers can no longer assume that any offer of a positive portion of the stake will result in acceptance of a proposal. Instead, she needs to account for the likelihood that a particular proposal will be accepted based on the preferences of her randomly (or perhaps more accurately haphazardly) assigned responder. The payoff for a proposal is then viewed best as an expected value based on the likelihood of rejection by a responder. The proposer might also reasonably account for the variability in outcomes to a proposal, as well as their expected value. For example, a proposal of $0-100$ that has a $50 \%$ probability of 
acceptance has the same expected monetary value to proposer as a 50-50 proposal which is certain to be accepted. Different proposers might value these quite differently, with some preferring the certainty of half the stake to the opportunity to receive all of the stake, while others hold the opposite valuation. Finally, the proposer must also weigh the value to her of the perception of her equitable or inequitable treatment of the responder. In the case of classroom play, the presence of witnesses with whom the players will spend the rest of the semester must affect their judgments. With these additional considerations we can create a model that plausibly explains not just the behavior of students in the classroom but also most of the broad array of behaviors observed in the Henrich study discussed at length above. (At least the Maragoli are still not reliably modeled.)

Equipped with such a model, a player can reasonably play the Ultimatum Game with the expectation that their preferences will be reflected in the outcome of the game. What is remarkable is that in the classroom and in most cultures around the world, this happens without such elaborate quantitative reasoning.

So, the issue is not irrational players, on the contrary we understand that players have subjective expectations of fairness that vary from individual to individual but display overall structure within a culture. The play in most cultures clearly reflects mutual understanding of this structure. Modeling this requires more sophisticated mathematics, including specifically probability, at least for the proposer. It is not unusual for adequately realistic models to demand learning new mathematics, and this game can serve to motivate and make concrete the value of understanding probability from a mathematical point of view. So, this has particular value in courses that include probability as part of the mathematical reasoning employed. That is not the case in the class being described here, however.

\section{Quantitative Literacy Is Not Simply Mathematical Reasoning}

A natural objection to this more elaborate model is that the assumptions do not reflect the thinking process that subjects experience when playing the game. In his readable exposition on practical applications of game theory, Rock, Paper, Scissors, Len Fisher (2008, 123-4) relates,

Researchers used functional magnetic resonance imaging to watch what was going on in the brains of the participants when they were accepting or rejecting offers. They found that a region of the brain known as the 'bilateral anterior insula,' which becomes very active during experiences of negative emotions such as anger and disgust, also becomes active when a low offer is received during the Ultimatum Game. By contrast, a brain area called the 'dorsolateral pre-frontal cortex,' or DLPFC, which is known to be involved in cognitive decision making, became very active when a high offer was made. 
It seems that in making decisions while playing the Ultimatum Game, the player is mediating between emotions, or at least deciding in light of activity in emotional centers of the brain.

Thus, play of the Ultimatum Game typically involves both emotional response and "cognitive decision making." It is important to acknowledge that in the vast majority of cases, quantitative literacy is exercised in just such a setting, with the need for flexible and creative application of mathematical competency, and temper that executive function with emotional motivations. Students should recognize that decisions that they face typically do not involve just quantitative considerations but also emotional ones, just like the decisions in the Ultimatum Game. And just as in real life, excessive reliance on either the rational or the emotional aspect of a decision might lead to outcomes that the player would regret. In his classic collection of poetic fables, The Prophet, Khalil Gibran $(1923,57)$ speaks of the roles of reason and emotion (passion), in a way that memorably makes this point: "Your reason and your passion are the rudder and the sails of your seafaring soul. If either your sails or your rudder be broken, you can but toss and drift, or else be held at a standstill in mid-seas."

The game serves as an object lesson for another little-appreciated dimension of quantitative literacy, captured by Peter Ewell's (2001, 37) observation, “... literacies are for the most part practiced invisibly and subconsciously ... not pulled out selectively and applied deliberately ...." We do not generally consciously apply mathematical reasoning in decision-making situations where a mathematical model is valuable. Still, when we have a flexible and extensible understanding of mathematics, it aids us "invisibly and subconsciously." When we do apply mathematical thinking, as we did in the previous subsection, it is typically after the heat of the moment. Sometimes this leads to regret and second-guessing, but it can often help us clarify our understanding of the situation and aid us in the heat of a similar moment in the future.

Beyond this, it is an important object lesson that useful mathematical models typically do not capture reality fully. They are approximations that offer insight but should not be trusted without limit.

One critical feature of the game as played in Henrich et al. (2006) and the author's classroom is the way the responder's strategy is determined. The responder is asked to consider each of the eleven possible proposals in turn and actively choose which ones are acceptable. This is a radically different decision process than simply responding at the moment to the proposal that is made. However, if the goal is to know the proposer's entire strategy, some such procedure is essential. In practice, students often regret the strategy they created when they learn its 
consequences. There are a number of reasons for this. Sometimes a responder decides to aggressively enforce equality, insisting on only a 50-50 split, only to lose when the proposer offers 40-60, or even more distressingly, 60-40. Other times the responder constructs some inscrutable model, for example, rejecting splits involving odd tens of percents of the stake (e.g., 10-90, 30-70, etc.), only to discover that they have rejected a 50-50 proposal. (Such strategies tend to come from students who lack confidence in their quantitative literacy.) Regardless, it is clear that most responders behave differently when forced to make a decision on a concrete proposal compared with considering all possible proposals in the abstract. This distinction is just an example of the way that quantitative literacy depends on context.

Context is crucially important in the Ultimatum Game, as it is in quantitative literacy more broadly. The experiments described earlier show that play depends sensitively on the precise rules and the instructions offered to the players. Reading Poundstone $(2010,120-1)$, students learn that when proposers earn the right to that role they tend to be less generous, and responders permit this. When the game is couched as a commercial transaction, with the proposer setting a price and the responder choosing to buy or not and the stake being divided between profit to the seller and savings to the buyer, once again proposers/sellers could be less generous with assent from responders/buyers. What is remarkable about these changes of context is that they were performed by both proposers and responders without conversation or coordination. Part of being acculturated to a time and place is being able to seamlessly change your valuation of proposals to different contexts that characterize that culture.

Just as subtle cultural clues adjust play of the game, it is cultural clues that alert us that the naive model of the Ultimatum Game ought to be treated with suspicion. More generally, part of quantitative literacy itself is the inclination to consider carefully a situation where quantitative information may pertain and judiciously apply not the most obvious but the most appropriate mathematical model. It is a weakness of our culture of math instruction that students so frequently and unreflectively apply models that are mathematically facile but miss cultural conventions that require more careful reflection. We depend too much on "word problems" that are transparent representations of mathematical concepts under study but are fantastical in their representation of reality. ("Two trains leave a station" problems are an archetype that receives appropriate derision.) Clearly, students need to see more realistic problems and learn the rudiments of mathematical modeling so that they develop a better sense of the richness of the 
modeling process and its centrality to quantitative literacy. The Ultimatum Game activity offers just such an opportunity.

\section{Conclusion}

The Ultimatum Game offers concrete evidence that many students in the classroom, like humans more broadly, generally place a measurable monetary value on fair treatment, willingly making monetary sacrifices in order to enforce or abide by fairness norms. In the course described here, the students discuss the game briefly after play and then write their first entry in their semester's journal about their thoughts and feelings while playing the game. They read the start of Henrich et al.(2006), a passage from Poundstone (2010, Chaps. 18-20), and an article by evolutionary biologist Olivia Judson (2007) that makes an argument for the evolutionary origin of altruism for a popular audience. The students have the option to choose a topic for their first major assignment of the semester from this collection. That paper is a developed personal reflection on readings and experiences from the course. A significant minority of students choose to make their topic from this initial segment of the class, and it generally serves them well. Their success seems to derive from the rich learning experience offered; for example, from it we learn that conceptions of fair treatment vary considerably from culture to culture, but cultures that have no fairness norms are extremely rare and apparently lack cohesion beyond the family.

Existence of these norms informs our understanding of social justice and allows us to see that creation of a society in which members generally believe that they are being treated fairly requires awareness of the variety of norms that different cultures within that society observe. The presence of fairness norms found nearly universally in a wide variety of cultures, their persistence when the size of the stake varies, and their sensitivity to context all suggest that fairness norms are central to a society's ability to prosper in the face of adversity. The Ultimatum Game offers evidence for the desire for fair treatment being at the foundation of social life and so suggests the importance of a coherent understanding of fair treatment in a society that can survive and thrive.

Modeling the Ultimatum Game in a way that incorporates the variety of fairness norms is more involved than the naive model offered in the introduction, but many people seem able to identify and apply the model using their own culture's norms without working out a mathematical model. This ability displays many of the characteristic features of quantitative literacy and demonstrates why it is so difficult to teach. Applying quantitative literacy to the Ultimatum Game is 
critical to revealing its insights into the foundations of social justice or fair treatment of fellow members of society and establishing a robust social order.

Thus, the Ultimatum Game offers a connection between quantitative literacy and social justice and provides a surprisingly nuanced view of their significance, both individually and collectively. Study of the game, including naïve playing of it followed by reading and writing about it, helps students draw these connections and experience these nuances.

\section{Acknowledgements}

The author is grateful for the inspiration and assistance of students, Chris Ruebeck and other colleagues, anonymous peer reviewers, and the editors in greatly improving this article.

\section{References}

Andersen, Steffen, Seda Ertaç, Uri Gneezy, Moshe Hoffman, and John A List. 2011. "Stakes Matter in Ultimatum Games." American Economic Review 101(7): 3427-39. https://doi.org/10.1257/aer.101.7.3427

Cameron, Lisa A. 1999. "Raising the Stakes in the Ultimatum Game: Experimental Evidence from Indonesia.” Economic Inquiry 37(1): 47-59. https://doi.org/10.1111/j.1465-7295.1999.tb01415.x

Candelo, Natalia, Catherine Eckel, and Cathleen Johnson. 2019. "The Proposer's Behavior in the Ultimatum Game in 11 Mexican Villages." Economics Letters 177(April): 5-8. https://doi.org/10.1016/j.econlet.2019.01.006

Cárdenas, Juan-Camilo. 2014. "Social Preferences among the People of Sanquianga in Colombia." In Experimenting with Social Norms: Fairness and Punishment in Cross-Cultural Perspective, edited by Jean Ensminger and Joseph Henrich, 391-420. New York: Russell Sage

Foundation. https://doi.org/10.2139/ssrn.1480354

Erickson, Patricia E. 2003. "Social Justice." In Encyclopedia of Community:

From the Village to the Virtual World, edited by Karen Christensen and David Levinson, 1309-14. Los Angeles: Sage Publishing.

Ewell, Peter T. 2001. "Numeracy, Mathematics and General Education: An Interview with Peter T. Ewell." In Mathematics and Democracy, edited by Lynn A. Steen, 37-48. Princeton: National Council on Education the Disciplines \& Woodrow Wilson National Fellowship Foundation.

Fisher, Len. 2008. Rock, Paper, Scissors: Game Theory in Everyday Life. New York: Basic Books. 
Gibran, Khalil. 1923. The Prophet. New York: Alfred A. Knopf. https://www.gutenberg.org/files/58585/58585-h/58585-h.htm

Gillman, Rick, and David Housman. 2009. Models of Conflict and Cooperation. Providence: American Mathematical Society. https://doi.org/10.1090/mbk/065

Güth, Werner, Rolf Schmittberger, and Bernd Schwarze. 1982. "An Experimental Analysis of Ultimatum Bargaining." Journal of Economic Behavior \& Organization 3(4): 367-388. https://doi.org/10.1016/0167-2681(82)90011-7

Halevy, Yoram, and Michael Peters. 2007. Is the Ultimatum Game the Ultimate Experiment? Midwest Economic Theory Meeting. Ann Arbor, Michigan, November. https://editorialexpress.com/conference/mwetfall2007/program/mwetfall2007 .html

Henrich, Joseph. 2000. "Does Culture Matter in Economic Behavior? Ultimatum Game Bargaining among the Machiguenga of the Peruvian Amazon." American Economic Review 90(4): 973-979. https://doi.org/10.1257/aer.90.4.973

Henrich, Joseph, Richard McElreath, Abigail Barr, Jean Ensminger, Clark Barrett, Alexander Bolyanatz, Juan Camilo Cardenas, Michael Gurven, Edwins Gwako, Natalie Henrich, Carolyn Lesorogol, Frank Marlowe, David Tracer, and John Ziker 2006. "Costly Punishment across Human Societies.” Science 312(5781): 1767-1770. https://doi.org/10.1126/science.1127333.

Judson, Olivia. 2007. "The Selfless Gene.” The Atlantic Monthly (October). Accessed October 2018. https://www.theatlantic.com/magazine/archive/2007/10/the-selflessgene/306196/

Nowak, Martin A, Karen M Page, and Karl Sigmund. 2000. "Fairness Versus Reason in the Ultimatum Game." Science 289(5485): 1773-1775. https://doi.org/10.1126/science.289.5485.1773

Poundstone, William. 2010. Priceless: The Myth of Fair Value (And How to Take Advantage of it). New York: Hill / Wang.

Price, Thomas. 1955. "Saints and Spirit: A Study of Differential Acculturation in Colombian Negro Communities.” Doctoral dissertation, Northwestern University.

Schecter, Stephen, and Herbert Gintis. 2016. Game Theory in Action. Princeton and Oxford: Princeton University Press. https://doi.org/10.2307/j.ctvcszzvz 
Slonim, Robert, and Alvin E Roth. 1998. "Learning in High Stakes Ultimatum games: An Experiment in the Slovak Republic." Econometrica 6(3): 569596. https://doi.org/10.2307/2998575

Webber, Wendy. 2017. Give Me Your Camera: Understanding Gift Giving in Ghana. Weblog, February. Accessed February 3, 2017.

https://appliedsentience.com/2017/02/03/give-me-your-cameraunderstanding-gift-giving-in-ghana/ 